\title{
BMJ Open Drug approval status and recommendations for listing on public formularies: a Canadian cohort analysis
}

\author{
Joel Lexchin
}

To cite: Lexchin J. Drug approval status and recommendations for listing on public formularies: a Canadian cohort analysis. BMJ Open 2017;7:e018372. doi:10.1136/ bmjopen-2017-018372

- Prepublication history for this paper is available online. To view these files please visit the journal online (http://dx.doi. org/10.1136/bmjopen-2017018372).

Received 26 June 2017 Revised 23 August 2017 Accepted 20 September 2017

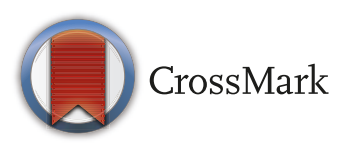

School of Health Policy \& Management, York University, Toronto, Ontario, Canada

Correspondence to Dr Joel Lexchin; jlexchin@yorku.ca

\begin{abstract}
Objectives Investigate if the recommendations by the Common Drug Review (CDR) and the pan-Canadian Oncology Drug Review (pCODR) to provincial, territorial and federal drug plans about whether to list non-oncology and oncology drug-indication combinations on their formularies are associated with whether the drug-indication combination was approved via the standard evidence pathway or the Notice of Compliance with conditions (NOC/c-limited evidence) pathway.

Design Cohort study.

Data sources Websites of the CDR and pCODR up to the end of 31 March 2017; journal articles evaluating drugs approved through the NOC/c pathway, the NOC database, the NOC/c website and the Summary Basis of Decision website.

Interventions Recommendations by the CDR and pCODR. Primary and secondary outcome measures Analysis of the percent of drugs receiving positive listing recommendations from CDR and pCODR depending on the pathway used to approve the drug.

Results There were 310 recommendations for drugindication combinations from the CDR and 79 from the pCODR. There was a statistically significant difference in the number of drug-indication combinations that received a list versus do not list recommendation from the CDR for those approved through the standard pathway compared with those approved through the NOC/c pathway $(p=0.0407)$. A similar analysis for recommendations from the pCODR was not statistically significant.

Conclusion For non-oncology drug-indication combinations, the type of review appears to influence the recommendation regarding listing on public formularies. This difference may reflect the level of evidence about the efficacy and safety of the drug indication at the time the recommendation was made.
\end{abstract}

\section{INTRODUCTION}

Health Canada is the branch of the Canadian government that, among other functions, is in charge of approving new drugs for marketing and subsequently monitoring their ongoing benefit to harm ratio. Like other major regulators, Health Canada approves drugs based on data about safety and efficacy. Once drugs are allowed on the market, two review processes administered by

\section{Strengths and limitations of this study}

Compares recommendations for formulary inclusion for oncology and non-oncology drugs.

- Evaluates whether recommendations are associated with whether drugs were approved via the standard evidence pathway or the Notice of Compliance with conditions (NOC/c; limited evidence) pathway.

- Small number of drug-indication combinations approved through the NOC/C pathway means that statistical significance can be influenced by small additions or subtractions.

the Canadian health technology assessment agency, the Canadian Agency for Drugs and Technology in Health (CADTH), assess the price and cost-effectiveness of medications for public formulary decision-making. Since 2003, the Common Drug Review (CDR) has been providing Canadian provincial (except for Quebec), territorial and federal public drug plans with recommendations about whether to list products on their formularies. Starting in 2011, the CDR was supplemented by the pan-Canadian Oncology Drug Review (pCODR), also part of CADTH, that provides the same advice regarding oncology medications. Both agencies accept applications from manufacturers and drug plans and then use expert panels $^{12}$ that consider the clinical evidence, plus input from patients, manufacturers and clinicians in making their recommendations about whether the plans should list drugs for specific indications.

However, the amount of clinical evidence that is available to these expert panels is not the same for all indications. The usual pathway to get a new active substance (NAS-a molecule never marketed before in Canada in any form, the equivalent term used by the European Medicines Agency is 'new chemical entity') or a new indication for an existing product approved for marketing in Canada is for the pharmaceutical company involved to file a New Drug Submission (NDS) including 
preclinical and clinical scientific information about the product's safety, efficacy and quality and information about its claimed therapeutic value, conditions for use and side effects. ${ }^{3}$ The key clinical evidence establishing the safety and efficacy of the new drug comes from the pivotal trials that Health Canada defines 'as trials of high scientific quality, which provide the basic evidence to determine the efficacy, properties, and conditions of use of the drug' ${ }^{4}$ Health Canada then has up to 300 days (standard review) or 180 days (priority review) to review the NDS and decide about whether to approve the drug or in the parlance of the agency issue a Notice of Compliance (NOC equals 'market authorisation' in Europe). Drugs approved under both the standard and priority approval pathways have completed all of the necessary trials, that is, up to and including phase III trials, and for the purposes of this study, they are collectively referred to as the standard approval pathway.

A second approval mechanism is the NOC with conditions $(\mathrm{NOC} / \mathrm{c}$ ) pathway which considers new drug submissions where the evidence is not complete. (The equivalent term in Europe is 'conditional approval'.) The goal of the NOC/c pathway is to 'provide patients suffering from serious, life threatening or severely debilitating diseases or conditions with earlier access to promising new drugs' where surrogate markers suggest that these new products offer "effective treatment, prevention or diagnosis of a disease or condition for which no drug is presently marketed in Canada or significantly improved efficacy or significantly diminished risk over existing therapies, ${ }^{5}$ (In the case of cancer, a surrogate outcome might be a shrinkage in tumour size or a longer time until the cancer recurs). Besides data based only on trials with surrogate markers, other instances where an NOC/c might be used are for NAS with phase II trials that require confirmation with phase III trials or NAS with a single small to moderately sized phase III trial that requires confirmation of either the efficacy or safety of the agent under question. ${ }^{6}$ In return, for NOC/c status, companies sign a Letter of Undertaking to complete confirmatory clinical studies, that is, studies that definitively establish efficacy, and submit the results of these to Health Canada. Should these postmarket trials not provide sufficient evidence of clinical benefit, the NOC/c could be revoked and the product removed from the market ${ }^{7}$ or an indication rescinded.

This study was undertaken to examine whether the level of evidence available to CDR and pCODR is associated with their recommendations about listing drugs on public formularies.

\section{METHODS}

\section{Construction of list of CDR and pCODR recommendations}

All the CDR reports up to the end of March 2017 were accessed from the CDR website https://www.cadth. $\mathrm{ca} /$ about-cadth/what-we-do/products-services/cdr/ reports. Only reports with final recommendations were examined, and if there was more than one report for a drug-indication combination, the most recent one was used. Different formulations of the same drug (eg, an oral and intravenous form) were considered as separate products. The following information was extracted from each report: generic name, brand name, indication for which listing was being sought, date of approval, listing recommendation and date of the recommendation. CDR has four different types of recommendations-list, list with clinical criteria and/ or conditions, do not list at the submitted price, do not list. These were dichotomised into list (list+list with clinical criteria and/or conditions) and do not list (do not list at the submitted price+do not list). As a subanalysis, drug-indication combinations with a 'do not list at the submitted price recommendation' were considered as a list recommendation.

Similarly, the reports from pCODR were accessed from https://www.cadth.ca/pcodr/find-a-review and the same information extracted. pCODR issues three different types of recommendations-recommend, consider with conditions, do not recommend and these were dichotomised into list (recommend+consider with conditions) and do not list (do not recommend).

\section{Construction of list of drug-indication combinations with NOC/c approval}

A list of all the NOC/c issued for new drugs and new indications for existing drugs from the beginning of the programme until 31 March 2017 and information about the drug-indication combination was compiled from five sources: articles by Lexchin ${ }^{7}$ and $\mathrm{Law}^{8}$ that listed NOC/c and investigated whether they had been fulfilled, the NOC database (available at http://webprod5.hc-sc.gc.ca/ noc-ac/index-eng.jsp), the NOC/c website (http://www. hc-sc.gc.ca/dhp-mps/prodpharma/notices-avis/conditions/index-eng.php) and the Summary Basis of Decision website (http://www.hc-sc.gc.ca/dhp-mps/prodpharma/ sbd-smd/drug-med/index-eng.php) that since 1 January 2005 summarises the clinical information used in making a decision to approve a new product. For each drug-indication combination, the following information was extracted: generic name, brand name, indication, date of $\mathrm{NOC} / \mathrm{c}$ and, if appropriate, date NOC/c fulfilled.

\section{Data analysis}

Information from the CDR and pCODR reports was matched with information about products with an $\mathrm{NOC} / \mathrm{c}$ based on generic name, indication and date of approval to determine which products considered by CDR and pCODR had been approved under the NOC/c pathway. Recommendations (list, do not list) from CDR and pCODR for drug-indication combinations approved through the standard pathway versus the NOC/c pathway were compared separately and together, that is, the CDR and pCODR recommendations were combined, using a $\chi^{2}$ analysis and Fisher's exact test to account for small sample sizes. A p 
value $<0.05$ was considered significant. All analyses were done with Prism V.7.0 for Mac (Graphpad software, 2016).

Only publicly available data were used and therefore ethics approval was not necessary.

\section{RESULTS}

There was a total of 389 reports on drug-indication combinations from the CDR and pCODR, 310 from CDR (247 drugs, range 1 to 6 indications per drug) and 79 from pCODR (59 drugs, range 1 to 5 indications per drug). When the recommendations of CDR and pCODR were combined and a comparison was done between drugs approved through the standard and NOC/c pathways, there was no statistically significant difference depending on the pathway $\left(\chi^{2}, \mathrm{p}=0.3119\right.$, data not shown) (see online Supplementary File at http://datadryad.org for the complete data set).

Of the 310 CDR reports, 295 (95.2\%) indications went through the standard approval pathway (177 list and 118 do not list recommendations) and $15(4.8 \%)$ went through the NOC/c pathway (5 list and 10 do not list recommendations). There was a statistically significant difference in the number of drug-indication combinations that received a list versus do not list recommendation for those approved through the standard pathway compared with those approved through the NOC/c pathway $\left(\chi^{2}, p=0.0407\right)$ (table 1$)$. There were nine 'do not list at the submitted price' recommendations for drugs approved through the standard pathway. When these were put into the list category, the results did not change. The NOC/c was fulfilled in 9 of the 15 cases but that fulfilment only came before the CDR recommendation on one occasion.

Of the 79 pCODR reports, $63(79.7 \%)$ indications went through the standard approval pathway (52 list and 11 do not list recommendations) and 16 (20.3\%) went through the NOC/c pathway (12 list and 4 do not list recommendations). There was no statistically significant difference in the number of drug-indication combinations that received a list versus do not list recommendation for those approved through the standard pathway compared with those approved through the NOC/c pathway (Fisher's exact test, $\mathrm{p}=0.4899$ ) (table 2). The NOC/c was fulfilled in 2 of the 16 cases but in neither case did that fulfilment happen before the pCODR recommendation.

Table 1 Recommendations from CDR for drug-indication combinations approved through the standard pathway versus the NOC/c pathway

\begin{tabular}{lrl}
\hline & List & Do not list \\
\hline Standard pathway & 177 & 118 \\
NOC/c pathway & 5 & 10 \\
\hline
\end{tabular}

$\chi^{2}, p=0.0407$.

CDR, Common Drug Review; NOC/c, Notice of Compliance with conditions.
Table 2 Recommendations from pCODR for drugindication combinations approved through the standard pathway versus the NOC/C pathway

\begin{tabular}{lll}
\hline & List & Do not list \\
\hline Standard pathway & 52 & 11 \\
NOC/c pathway & 12 & 4 \\
\hline
\end{tabular}

Fisher's exact test, $\mathrm{p}=0.4899$.

$\mathrm{NOC} / \mathrm{c}$, Notice of Compliance with conditions; pCODR, panCanadian oncology drug review.

\section{DISCUSSION}

When the recommendations of $\mathrm{CDR}$ and pCODR are combined, the approval pathway is not associated with whether a recommendation to list or not to list is made. However, analysed separately, the standard approval pathway is associated with positive listing recommendations for non-oncology drugs that have been reviewed by the CDR, although not for oncology drugs reviewed by the pCODR.

In almost all cases, both the CDR and pCODR are considering NOC/c-approved drugs before their conditions have been fulfilled and therefore before there is definitive evidence about their efficacy and safety. In this situation, the lesser amount of evidence may affect recommendations to public drug plans about whether to list non-oncology drugs for particular indications. The difference in the results for oncology drugs versus drugs for other indications may be because the pCODR is willing to accept more uncertainty due to the serious nature of cancer, but this interpretation of the results is speculative and would require verification in future research.

When drugs are newly marketed, even if they have been approved based on a complete set of premarket studies, knowledge about them is still limited, especially with respect to safety. Almost $20 \%$ of drugs will eventually acquire a new serious safety warning ${ }^{9}$ and from 1990 to 2009, between $4 \%$ and $5 \%$ approved in a 5-year period were eventually removed from the Canadian market because of safety reasons. ${ }^{10}$ Just under $50 \%$ of new drug approvals in Canada are based on trials with surrogate end points ${ }^{11}$ and surrogate outcomes are often not a reliable indicator of the true efficacy/effectiveness of new drugs. ${ }^{12}$ The uncertainty about safety and efficacy can complicate listing decisions about all new drugs, but even more so for those with an NOC/c approval.

In the case of CDR, having an arms-length agency to make recommendations based on a cost-effectiveness basis can be viewed in a positive light, by creating a barrier to listing when the data about the drugs are insufficient or when the benefits are not commensurate with the costs. Not only is there a saving to the public purse, but the CDR recommendations may give doctors and patients the confidence that the gatekeeper function is working appropriately. On the other hand, patients and doctors may feel frustrated about the reticence of the CDR to recommend listing drugs approved 
through the NOC/c pathway as they see this as denying them needed therapy. Waiting for more information may mean delaying a decision for almost 4 years, the mean length of time that it takes to fulfil a NOC $/ \mathrm{c},{ }^{13}$ and some drugs approved under the NOC/c pathway have been on the market for over 10 years without fulfilling their conditions. $^{8}$

One attempt to cope with this degree of uncertainty has been to use coverage with evidence development (CED) whereby funding is conditional until more evidence is generated and if the evidence is negative, funding could be completely withdrawn. However, CED approaches are often not successful because the political and economic relations involved in these schemes are often ignored. As a result, tensions emerge in multiple areas such as how evidence should be interpreted, the roles and responsibilities of the various stakeholders and who is responsible for funding decisions. ${ }^{14}$ Another approach is performance-based agreements whereby the amount paid for a drug is tied to the health outcomes achieved. The amount is retroactively adjusted through rebates from the drug manufacturer to the payer if the drug does not produce the expected health outcomes either because it is less effective or more unsafe than was initially believed. ${ }^{15} 16$ However, in the case of this type of agreement for multiple sclerosis (MS) drugs in the UK, the patients on the MS drugs fared far worse than those on placebo. According to the trial agreement, this result should have triggered a reduction in the price paid for the two drugs being examined. This reduction did not happen; instead, a report claimed that the scientific advisory group considered that it was premature at this stage to reach any decision about re-pricing the drugs without further follow-up and analyses'. ${ }^{17}$

There are a number of limitations to this study. Data extraction was done by a single person with the resultant possibility of a random bias. However, the type of data used was straightforward, for example, dates and drug names, which minimises the chance for biased interpretation. The small number of drug-indication combinations approved through the NOC/c pathway also means that statistical significance can be influenced by small additions or subtractions and therefore as more use is made of this pathway results that were non-significant may become significant and vice versa.

This study should be repeated in the future when a larger number of products have gone through the NOC/c pathway to investigate whether the results are stable. In addition, research could examine not just whether CDR and pCODR recommendations are associated with the available level of evidence, but whether the level of evidence affects provincial listing decisions.

Limited evidence can, in some cases, influence reimbursement decisions by Canadian agencies. Both the US Food and Drug Administration and the European Medicines Agency are moving in the direction of allowing products on the market with less evidence. ${ }^{18} 19$ Should Health Canada follow suit, then both the CDR and pCODR will be faced with making recommendations on more products where safety and efficacy are uncertain.

In the face of similar concerns about prices and clinical effectiveness, in 2011, the Australian government and the industry association, Medicines Australia, introduced a framework agreement for an outcomes-based managed entry scheme (MES). Under a MES, the Australian health technology assessment agency, the Pharmaceutical Benefits Advisory Committee (PBAC), could recommend the listing of a new medicine with a high clinical need at a price justified by the existing evidence, pending the availability of more conclusive evidence of cost-effectiveness. ${ }^{20} 21$ Although not formally stated in the framework agreement, the expectation is that new evidence would be in the form of randomised clinical trials with hard clinical outcomes and that superiority trials will be more acceptable to the PBAC. ${ }^{21}$ Currently, CDR and pCODR can recommend not listing at the submitted price, but if the number of NOC/c-approved products increase, Health Canada should consider expanding the mandate of the CDR and pCODR to include recommendations for listing drugs under a managed entry scheme modelled on the one used in Australia.

Contributors $\mathrm{JL}$ came up with the idea for this study, gathered and analysed the data and wrote the manuscript.

Funding There was no funding involved in the research or writing of this article.

Competing interests In 2015-2017, JL received payment from two non-profit organisations for being a consultant on a project looking at indication-based prescribing and a second looking at which drugs should be distributed free of charge by general practitioners. In 2015 , he received payment from a for-profit organisation for being on a panel that discussed expanding drug insurance in Canada. He is on the Foundation Board of Health Action International.

Provenance and peer review Not commissioned; externally peer reviewed.

Data sharing statement Extra data can be accessed via the Dryad data repository at http://datadryad.org with the doi:10.5061/dryad.tv138.

Open Access This is an Open Access article distributed in accordance with the Creative Commons Attribution Non Commercial (CC BY-NC 4.0) license, which permits others to distribute, remix, adapt, build upon this work non-commercially, and license their derivative works on different terms, provided the original work is properly cited and the use is non-commercial. See: http://creativecommons.org/ licenses/by-nc/4.0/

(c) Article author(s) (or their employer(s) unless otherwise stated in the text of the article) 2017. All rights reserved. No commercial use is permitted unless otherwise expressly granted.

\section{REFERENCES}

1. CADTH pan-Canadian Oncology Drug Review: Process in Brief Process in brief: secondary CADTH pan-Canadian oncology drug review, 2017. https://www.cadth.ca/pcodr/process-in-brief.

2. CADTH Common Drug Review. Procedure for the CADTH Common Drug Review. Secondary procedure for the CADTH common drug review, 2014. https://www.cadth.ca/sites/default/files/cdr/process/ Procedure_for_CADTH_CDR.pdf.

3. Health Canada: Health Products and Food Branch. Access to therapeutic products: the regulatory process in Canada. Ottawa, 2006.

4. Health Canada. Preparation of human new drugsubmissions. Secondary preparation of human new drug submissions, 2009. http://www.hc-sc.gc.ca/dhp-mps/prodpharma/applic-demande/ guide-ld/newdrug-drognouv/prephum-eng.php.

5. Health Canada. Notice of compliance with conditions (NOC/c). Ottawa, 2002.

6. Health Products and Food Branch. Guidance document: notice of compliance with conditions (NOC/c), 2011. 
7. Lexchin J. Notice of compliance with conditions: a policy in limbo. Healthc Policy 2007;2:114-22.

8. Law MR. The characteristics and fulfillment of conditional prescription drug approvals in Canada. Health Policy 2014;116:154-61.

9. Lexchin J. New drugs and safety: what happened to new active substances approved in Canada between 1995 and 2010? Arch Intern Med 2012;172:1680-1.

10. Lexchin J. How safe are new drugs? Market withdrawal of drugs approved in Canada between 1990 and 2009. Open Medicine 2013;8:e14-9.

11. Lexchin J, Ahmed T. Postmarket safety of drugs approved by Health Canada on the basis of clinical and surrogate outcomes: a cohort study. CMAJ Open 2015;3:E286-91.

12. Svensson S, Menkes DB, Lexchin J. Surrogate outcomes in clinical trials: a cautionary tale. JAMA Intern Med 2013;173:611-2.

13. Lexchin J. Publication of confirmatory studies required by health Canada for drugs approved under a notice of compliance with conditions: a cohort study. CMAJ Open 2017;5:E295-300.
14. Bishop D, Lexchin J. Politics and its intersection with coverage with evidence development: a qualitative analysis from expert interviews. BMC Health Serv Res 2013;13:88.

15. Docteur E, Lopert R. Payment policies to manage pharmaceutical costs: insights from other countries, 2017.

16. Chapman S, Reeve E, Rajaratnam G, et al. Setting up an outcomes guarantee for pharmaceuticals: new approach to risk sharing in primary care. BMJ 2003;326:707-9.

17. Raftery J. Multiple sclerosis risk sharing scheme: a costly failure. BMJ 2010;340:c1672-4.

18. Kesselheim AS, Avorn J. New "21st Century Cures" legislation:speed and ease vs science. JAMA 2017;317:581-2.

19. Davis C, Lexchin J, Jefferson T, et al. "Adaptive pathways" to drug authorisation: adapting to industry? BMJ 2016;354:i4437.

20. Vitry A, Roughead E. Managed entry agreements for pharmaceuticals in Australia. Health Policy 2014;117:345-52.

21. Wonder M, Backhouse ME, Sullivan SD. Australian managed entry scheme: a new manageable process for the reimbursement of new medicines? Value Health 2012;15:586-90. 